\title{
Condiloma Acuminado em Crianças e Adolescentes
}

\author{
Condyloma Acuminatum in Children and Adolescents
}

Marta Francis Benevides Rehme, Newton Sérgio de Carvalho, Mauro Fernando Kürten Ihlenfeld, Ana Carolina Silva Chuery

\begin{abstract}
RESUMO
Objetivo: analisar os fatores epidemiológicos, manifestações clínicas e forma de tratamento da infecção pelo papilomavírus.

Métodos: todos os casos de condiloma acuminado em crianças e adolescentes atendidas no periodo de 1990 a 1995 no Ambulatório de Ginecologia Infanto-Puberal foram revisados, tendo sido coletados dados referentes a idade, manifestações clínicas, local das lesões, formas de transmissão e tratamento.

Resultados: entre os 18 casos estudados, a média de idade foi de 6 anos e 11 meses (variando de 2 a 15 anos). A manifestação clínica mais comum foi a presença de verrugas $(61,1 \%)$. As lesões eram localizadas na região vulvoperineal em $44,4 \%$ das pacientes, sendo que lesões perianais e vulvares foram observadas em 27,8\% e 22,2\% dos casos, respectivamente. Não foi possível confirmar a ocorrência de abuso sexual nem de lesões condilomatosas nos pais em 66,7\% dos casos. Provável abuso sexual (não-confirmado) foi relatado em 2 casos. A terapêutica básica foi a cauterização química.

Conclusões: o abuso sexual em crianças e adolescentes com condiloma acuminado deve ser investigado, apesar da existência de outras formas de transmissão, incluindo auto ou heteroinoculação. As formas de apresentação na idade jovem diferem das do adulto, sendo necessária uma terapêutica adequada a essa população.
\end{abstract}

PALAVRAS-CHAVE: HPV. Condiloma acuminado. Abuso sexual. Doenças sexualmente transmissíveis. Infância e adolescência.

\section{Introdução}

A freqüência de crianças infectadas pelos papilomavírus humano (HPV) tem aumentado e parece ser relacionado ao aumento da incidência

Ambulatório de Ginecologia Infanto-Puberal - Serviço de Ginecologia do Departamento de Tocoginecologia da Universidade Federal do Paraná - UFPR

Correspondência:

Marta F Benevides Rehme

Av. Água Verde, 1475 apto 1701 - Bairro Água Verde

80620-200 Curitiba - PR

Fone: (041) 243-6065 de condiloma em adultos ${ }^{1}$. A avaliação médica e o manejo da infecção pelo HPV em crianças é complicada pelo longo período de latência do vírus, diferentes modos de transmissão e ausência de um regime terapêutico único e eficaz ${ }^{2,3,14}$. As crianças infectadas são potencialmente de risco para o desenvolvimento de neoplasia intra-epitelial cervical (NIC), neoplasia intra-epitelial vulvar (NIV) e neoplasia intra-epitelial vaginal (NIVA).

$\mathrm{O}$ abuso sexual tem sido considerado o principal modo de transmissão e alguns autores consideram que a simples presença de condiloma em crianças pode ser um indicativo de que esteja 
havendo abuso sexual ${ }^{16}$. A incidência de abuso sexual em crianças portadoras de HPV tem sido observada em 10 a $90 \%$ dos casos, e esta discrepância se deve à maneira como a investigação do abuso sexual foi realizada ${ }^{13}$.

O objetivo deste trabalho foi de analisar os fatores epidemiológicos, manifestações clínicas e forma de tratamento da infecção pelo papilomavirus e verificação da ocorrência de infecção no país.

\section{Pacientes e Métodos}

Procedemos à avaliação dos prontuários de 18 crianças e adolescentes portadoras de condiloma acuminado, atendidas no Ambulatório de Ginecologia Infanto-Puberal do Serviço de Ginecologia do Departamento de Tocoginecologia da UFPR no período de 1990 a 1995. Foram tabulados dados referentes a: idade, manifestação clínica, sítio da lesão, possível modo de transmissão e tratamento.

\section{Resultados}

A idade das pacientes variou de 2 a 15 anos, com média de 6 anos e 11 meses. Metade tinha entre 2 e 5 anos e 33,3\% entre 6 e 10 anos (Tabela 1).

Tabela 1 - Condiloma acuminado em crianças e adolescentes. Distribuição dos casos segundo a faixa etária.

\begin{tabular}{lcr}
\hline Idade & $\mathbf{N .}^{\mathbf{0}}$ casos & $\boldsymbol{\%}$ \\
\hline $0-5$ & 9 & 50,0 \\
$6-10$ & 6 & 33,3 \\
$11-15$ & 3 & 16,7 \\
\hline Total & 18 & 100,0
\end{tabular}

A manifestação clínica predominante foi a presença de verrugas, encontradas em $61,1 \%$ das pacientes, estando associadas com corrimento e/ ou prurido vulvar em 11\% (Tabela 2). Em 44,4\% dos casos as lesões ocorreram na região vulvoperineal. Localizações isoladas em ânus e vulva foram observadas em $27,8 \%$ e $22,2 \%$ dos casos respectivamente (Tabela 3 ).

A terapêutica básica instituída foi a destruição química em 13 pacientes. Utilizou-se o ácido tricloroacético a 50\% (ATA 50\%) em 8 casos (em 1 caso associou-se o tratamento com alça diatérmica), e podofilina a $25 \%$ em 5 pacientes. Três pacientes foram submetidas a criocauterização e houve 2 casos de cura espontânea (Tabela 4).

Tabela 2 - Manifestação clínica (queixa e achados) em crianças e adolescentes portadoras de condiloma acuminado.

\begin{tabular}{lcr}
\hline Queixa principal & Casos & $\%$ \\
\hline Verruga & 11 & 61,1 \\
Corrimento e/ou prurido & 4 & 22,2 \\
Verruga + corrimento & 1 & 5,5 \\
Verruga + prurido & 1 & 5,5 \\
Mancha & 1 & 5,5 \\
Total & 18 & 100,0 \\
\hline
\end{tabular}

Tabela 3 - Distribuição dos casos de condiloma acuminado em crianças e adolescentes segundo sítio da lesão.

\begin{tabular}{lcr}
\hline Sítio da lesão & N. $^{\mathbf{0}}$ Casos & \% \\
\hline Vulvo-perineal & 8 & 44,4 \\
Perianal & 5 & 27,8 \\
Vulva & 4 & 22,2 \\
Periuretral & 1 & 5,5 \\
Total & 18 & 100,0 \\
\hline
\end{tabular}

Tabela 4 - Tipos de tratamento utilizados em crianças e adolescentes portadoras de condiloma acuminado.

\begin{tabular}{lcr}
\hline Tratamento & N. $^{\mathbf{0}}$ Casos & \% \\
\hline ATA 50\% & 7 & 38,9 \\
Podofilina 25\% & 5 & 27,8 \\
Criocauterização & 3 & 16,7 \\
ATA + Alça & 1 & 5,5 \\
Cura espontânea & 2 & 11,1 \\
Total & 18 & 100,0 \\
\hline
\end{tabular}

Para determinar a fonte de infecção utilizamos anamnese dirigida para pesquisa de abuso sexual. Foram detectados antecedentes familiares de infecção pelo HPV em 4 pacientes. Em 3 casos os pais eram portadores de verrugas genitais, e um irmão em 1 caso. História de abuso sexual foi relatada em apenas 2 casos e nenhuma delas foi confirmada. Os 12 casos restantes $(66,7 \%)$ 
negavam antecedentes familiares e história de abuso sexual (Tabela 5).

Tabela 5 - Fonte de infecção pelo papilomavírus em crianças e adolescentes.

\begin{tabular}{lcr}
\hline Fonte de infecção & N. $^{\mathbf{0}}$ casos & \% \\
\hline Irmãos & 1 & 5,5 \\
Pais & 3 & 16,7 \\
História de abuso sexual * & 2 & 11,1 \\
Desconhecido & 12 & 66,7 \\
\hline
\end{tabular}

* Não-confirmada

\section{Discussão}

Os serviços de atendimento a crianças e adolescentes têm relatado um grande número de lesões condilomatosas em meninas de 3 a 6 anos. Observamos entre nossos casos uma média de idade de 6 anos e 11 meses o que coincide com outros relatos de literatura ${ }^{6,14,15}$.

Embora na população adulta a infecção pelo HPV seja considerada quase exclusivamente uma doença sexualmente transmissivel, outros modos de transmissão podem ocorrer nas crianças como transmissão vertical, inoculação digital ou por meio de outras lesões, fomites ${ }^{1,4}$. A transmissão vertical é a que ocorre durante o trabalho de parto com transmissão direta da mãe para o recém-nascido, podendo se estender até o período perinatal. A presença de lesões em crianças com menos de 3 anos de idade sugere este modo de transmissão, vista que o período de latência estimado por alguns autores pode variar de 1 a 3 anos. Na maioria das crianças com mais de 3 anos de idade portadoras de verrugas ano-genitais têm sido encontradas evidências de abuso sexual. A confirmação depende de métodos de avaliação com acurácia variável o que leva a uma grande discrepância na prevalência do abuso, que pode variar de 10 e $90 \%^{2,3,5,9}$. Esta dificuldade, também encontramos entre nossas pacientes, visto que não se conseguiu determinar o modo de transmissão na maior parte dos casos. Observamos em nossos resultados que a incidência de abuso sexual no entanto, foi baixa, contrastando com a literatura. Mas, ocorrência de abuso sexual deve sempre ser levado em consideração e investigado.

Outro aspecto importante é que os tipos de vírus que causam papiloma no trato genital são o mesmo para crianças e adultos ${ }^{8}$. No trabalho de Obalek et $\mathrm{al}^{13}$, no qual se avaliaram crianças com idade entre 7 meses e 12 anos portadoras de verrugas anogenitais, foi constatado uma prevalência de $17,4 \%$ de HPV 2 (causadores da verruga comum) e 74\% de HPV 6. Este trabalho enfatiza que a auto-inoculação por parte de crianças portadoras de verrugas em mãos, ou heteroinoculação pode ser um modo de transmissão não-sexual ${ }^{13,17}$. A aquisição do HPV durante a infância e a adolescência não é uma causa imediata de morbidade grave, porém, há fortes evidências que a exposição precoce não somente precipite o desenvolvimento de cânceres anogenitais mas também aumente o risco para o câncer ${ }^{11}$.

A manifestação clínica mais freqüente é a presença de verrugas em sua maioria assintomáticas, sendo um achado casual durante troca de fraldas, banhos ou evidenciado pelo pediatra durante o exame físico. Outras queixas incluem dor, prurido e sangramento ${ }^{2,3}$. Estes dados coincidem com nossos achados, um vez que a presença de verrugas assintomáticas foi observada em $61 \%$ das pacientes.

O local mais comum do aparecimento do condiloma acuminado em crianças é a região perianal, podendo se estender até o canal anal. Particularmente nas meninas, pode-se encontrar lesões periuretrais, himenais e em fúrcula vaginal se apresentando de forma irregular ou como múltiplas pequenas pápulas, estendendo-se até grandes e pequenos lábios ${ }^{3,12}$.Observamos que em nossa casuística, o local predominante foi vulva e/ou períneo, totalizando 95\% dos achados. Em razão das limitações do exame ginecológico em meninas pré-puberes, o condiloma vaginal e cervical é raramente descrito em literatura ${ }^{3}$. No entanto, nos trabalhos em que se examinaram crianças que sofreram abuso sexual e foram submetidas a coleta de material de lavado vaginal, tem sido descrito achado de DNA de $\mathrm{HPV}^{7,12}$. O diagnóstico diferencial inclui condiloma plano da sífilis secundária, molusco contagioso, pênfigo benigno crônico, histiocitose X, neurofibromatose, rabdomiossarcoma (sarcoma botrióide).

O tratamento ideal do condiloma em crianças deveria ser de baixo custo, efetivo, atraumático e amplamente acessível e infelizmente ainda não é disponivel. Os métodos utilizados são a destruição química ou mecânica das lesões por meio de criocauterização, eletrocauterização ou alça diatérmica. Estes métodos apresentam o inconveniente de serem dolorosos e deixarem seqüelas. Para a destruição química tem sido descrito o uso de podofilina a $25 \%$, ATA com concentração entre 50 e $80 \%$, 5-fluorouracil creme $(5-\mathrm{FU})^{9}$.

Temos utilizado para o tratamento de infecções pelo HPV o ácido tricloroacético a 50\% (ATA 50\%), sendo a excisão cirúrgica pelo criocautério ou alça reservada a leões extensas. Embora a podofilina 25\% tenha sido utilizada como tratamento, temos reduzido seu uso por causa da absorção desproporcionalmente aumentada em crianças. Calcula-se que $1 \mathrm{~cm}^{2}$ de superfície de pele em uma criança de aproximadamente 3 anos 
de idade corresponde a uma superfície corporal de $3 \mathrm{~cm}^{2}$ de um adulto. Outro fator que restringe seu uso é a necessidade de remoção com banhos de assento após 4 horas da aplicação. Uma alternativa seria o uso de soluções diluídas de 5 a $15 \%{ }^{9}$.

A resolução espontânea foi observada em 2 casos após medidas locais de higiene prévias ao tratamento que seria instituído, confirmando dados de literatura, embora seja uma forma de evolução ocasional $^{10}$.

\section{SUMMARY}

Parpose: to analyze the epidemiologic factors, clinical manifestations and forms of treatment of infection with papiloma virus.

Method: all cases of condyloma acuminatum in children and adolescents assisted in the period from 1990 to 1995 in the Service of Children and Adolescent Gynecology were revised. We present the following data: age, diagnosis, clinical manifestations, sites of the lesions, transmission modes and treatment.

Results: the average age of the 18 studied cases, was 6 years and 11 months (ranging from 2 to 15 years). The most common clinical manifestation was the presence of warts (61.1\%). The lesions were located in the vulvoperineal area in $44.4 \%$ of the patients, and perianal and vulvar lesions were observed respectively in $27.8 \%$ and $22,2 \%$ of the cases. It was not possible to confirm the occurrence of sexual abuse or of condyloma lesions in the parents in $66.7 \%$ of the cases. Probable sexual abuse (not confirmed) was reported in 2 cases. The basic therapy was chemical cauterization.

Conclusions: sexual abuse in children and adolescents with condyloma acuminatum should be investigated in spite of the existence of other transmission ways including auto- or heteroinoculation. The presentation forms at young age differ from those in adults, and thus an appropriate therapy for this is necessary for this population.

KEY WORD: HPV. Condyloma acuminatum. Sexual abuse. Sexually transmitted disease. Childhood and adolescence.

\section{Referências}

1. Armstrong DK, Handley JM. Anogenital warts in prepubertal children: pathogenesis, HPV typing and management. Int J STD AIDS 1997; 8:78-81.

2. Emans SJH, Goldstein DP. Pediatric \& adolescent gynecology. $3^{\text {th }}$ ed Boston: Little Brown; 1990. p. 385.
3. Frasier LD. Human papillomavirus infections in children. Pediatr Ann 1994; 23:354-60.

4. Gross G. Condylomata acuminata in childhood - pointing to sexual abuse. Hautarzt 1992; 43:120-5.

5. Gutman LT, Herman-Giddens ME, Phelps WC. Transmission of human genital papillomavirus disease: comparison of data from adults and children. Pediatrics 1993; 91:31-8.

6. Gutman LT, St Claire KK, Everett VD, Ingram DL, Soper J, Johnston WW, et al. Cervical-vaginal and intraanal human papillomavirus infection of young girls with external genital warts. J Infect Dis 1994; 170:339-44.

7. Gutman LT, St Claire KK, Herman-Giddens ME, Johnston WW, Phelps WC. Evaluation of sexually abused and nonabused young girls for intravaginal human papillomavirus infection. Am $\mathbf{J}$ Dis Child 1992; 146:694-9.

8. Hanson RM, Glasson M, Mccrossin I, Rogers M, Rose B, Thompson C. Anogenital warts in childhood. Child Abuse Neg1 1989; 13:225-33.

9. Hicks RA. Empiric therapy of perianal lesions in a sexually abused child: medical and forensic implications. Pediatr Emerg Care 1993; 9:346-7.

10. Kraus SJ, Stone KM. Management of genital infection caused by human papillomavirus. Rev Infect Dis 1990; 12(Suppl 6):S620-32.

11. Moscicki AB. Genital HPV infections in children and adolescents. Obstet Gynecol Clin North Am 1996; 23:675-97.

12. Mulchahey KM. Sexually transmitted diseases in childhood. In: Sanfilippo JS, Muram D, Lee PA, Dewhurst J. Pediatric and adolescent gynecology. Philadelphia: Saunders; 1994. p. 321-35.

13. Obalek S, Misiewicz J, Jablonska S, Favre M, Orth G. Childhood condyloma acuminatum: association with genital and cutaneous human papillomaviruses. Pediatr Dermatol 1993; 10:101-6.

14. Paradisi M, Angelo C, Cianchini G, Mostaccioli S, Puddu P. I condilomi acuminati in età pediatrica: etiopatogenesi e revisioni della letteratura. Minerva Pediatr 1993; 45:341-6.

15. Paradisi M, Angelo C, Cianchini G, Mostaccioli S, Puddu P. La terapia dei condilomi acuminati in età pediatrica. Minerva Pediatr 1993; 45:419-24.

16. Wright CA, Taylor L, Cooper K. HPV typing of vulvovaginal condylomata in children. S Afr Med $\mathbf{J}$ 1995; 85: (10 Suppl): 1096-101.

17. Yell JA, Sinclair R, ManN S, Fleming K, Ryan TJ.. Human papillomavirus type 6-induced condylomata: an unusual complication of intertrigo. Br J Dermatol, 1993; 128:575-7. 